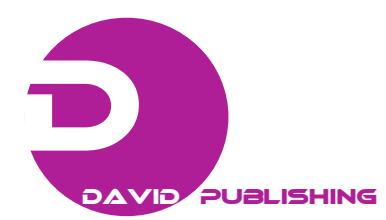

\title{
Effect of Vacuum Pre-cooling on Quality Properties of Broccoli after Harvest Compared with Other Conventional Cooling Methods
}

Tian Ding, Fen Liu, Xingqian Ye, Jianwei Zhou and Donghong Liu*

Department of Food Science and Nutrition, School of Biosystems Engineering and Food Science, Zhejiang University, Hangzhou, Zhejiang 310058, P.R. China

*Corresponding author's e-mail: dhliu@zju.edu.cn

\begin{abstract}
Vacuum pre-cooling technology has potential to be used in post-harvest storage of vegetables by removing field heat rapidly. In the current study, the efficiencies of different cooling methods (vacuum cooling, cooling water and cold room) and the quality properties of broccolis with pre-cooling treatments were compared. The results indicated that vacuum cooling treatment was the most effective method to extend the shelf life of post-harvest broccolis through the investigation of cooling rate, respiration rate, chlorophyll, vitamin C, reducing sugar, and sensory properties. Besides, by means of applying water-spraying on broccolis during vacuum cooling process, the weight loss was significantly reduced which was slightly higher than that resulted by the other two cooling methods and it did not negatively affect the quality of broccolis. Consequently, vacuum pre-cooling treatment can be applied on vegetables after harvest in the field to maintain the quality during transportation and storage. Also, the application of water-spraying in vacuum cooling technology can considerably reduce the negative influence caused by vacuum pre-cooling.
\end{abstract}

Key words: Vacuum pre-cooling, broccoli, post-harvest storage, cooling water. 